\title{
Colecta de germoplasma de higuerilla (Ricinus communis L.) en dos municipios de Arauca, Colombia
}

\author{
Collection of castor-oil plant germoplasm \\ (Ricinus communis L.) in two municipalities \\ of Arauca, Colombia
}

\section{Coleção de germoplasma (Ricinus communis L.) em dois municípios de Arauca, Colômbia}

\author{
Carlos Iván Cardozo Conde ${ }^{1}$ \& César Augusto Posada Tique ${ }^{2}$ \\ IIngeniero Agrónomo, Magister en Sistemas de Semillas, Doctor en Fitomejoramiento. ${ }^{2} Z o o t e c n i s t a, ~ M a g i s-$ \\ ter en Ciencias Agrarias (Producción Animal Tropical) y Doctor en Ciencias Agropecuarias (mejoramiento \\ genético vegetal y producción de cultivos \\ ${ }^{1}$ Facultad de Ciencias Agropecuarias. Universidad Nacional de Colombia, Sede Palmira, Colombia. ${ }^{2}$ Grupo \\ de Investigación en Recursos Fitogenéticos Neotropicales (GIRFIN) de la Universidad Nacional de Colombia, \\ Sede Palmira, Colombia \\ ํicardozoc@unal.edu.co, ${ }^{2} \underline{\text { capososadat@unal.edu.co }}$
}

\section{Resumen}

Ricinus communis L. conocido comúnmente como higuerilla es importante por su utilización en biocombustibles. Con el objetivo de conocer su estado actual se realizó, en 40 veredas de influencia de los complejos petroleros de Caricare (municipio de Arauquita) y Caño Limón (municipio de Arauca) en Arauca, Colombia, una colecta de semilla sexual entre diciembre del 2011 y enero del 2012 donde hace cinco años se estableció el cultivo. Entre las variables evaluadas se incluyeron datos de pasaporte como fuente de colecta, nombre local, relieve, tipo de suelo, entre otras. Para identificar los transectos y registrar datos como ubicación geográfica y altitud sobre el nivel del mar se utilizó un GPS Garmin map76CSx. Se realizó estadística descriptiva simple para identificar el conjunto de variables con mayor variación. Con las variables cualitativas de mayor importancia se realizó un análisis de tablas de contingencia y contraste con un nivel de significancia del $5 \%$, análisis de correspondencia múltiple (ACM) y de componentes principales (ACP) para variables cuantitativas y cualitativas, y se realizó un análisis de clasificación a partir de una matriz de similitud. Se encontró higuerilla en el 25\% de las veredas visitadas. Se colectaron 12 introducciones, cuatro de ellas en Caricare y ocho en Caño Limón. Aunque las condiciones ambientales resultan propicias para su cultivo, no existen cultivos comerciales en las localidades visitadas. La ausencia de comercialización del grano y de equipos para la extracción del aceite es el principal limitante. Se estableció un jardín con los materiales colectados con propósito de uso en investigación, mejoramiento genético y fomento. 
Palabras clave: biodiesel, Caricare, Caño Limón, higuerilla, tártago, Ricinus communis $L$.

\section{Abstract}

Ricinus communis L., commonly known as the castor-oil plant, is important for its use in biofuels production. With the objective of learning about its current status, in 40 villages influential to the oil complex of Caricare (municipality of Arauquita) and Caño Limón (municipality of Arauca) in Arauca, Colombia (where five years earlier the crop had been established), a collection of sexual seed was carried out between December 2011 and January 2012. The variables studied include passport data as a collection resource, local name, relief, and soil type among others. A Garmin map76CSx was used in order to identify the transects and record data such as geographic location and elevation. Simple descriptive statistics were used to identify the variables of greatest variation. Using the qualitative variables of greatest importance, a contingency table analysis with a significance level of $5 \%$, a multiple correspondence analysis (MCA) and principal component analysis (PCA) were performed for quantitative and qualitative variables, in addition to a classification analysis through a similarity matrix. The castor-oil plant was found in $25 \%$ of the villages visited. 12 introductions were collected, four from Caricare and eight from Caño Limón. Although the environmental conditions were favorable for its cultivation, there are no castor-oil plant crops in the locations visited. The absence of grain commercialization and oil extraction equipment is the main limiting factor. A garden was established using collected materials for the purpose of research, breeding and propagation.

Key-words: biodiesel, Caricare, Caño Limón, castor oil plant, spurge, Ricinus communis $L$.

\section{Resumo}

Ricinus communis L. habitualmente conhecida como mamona ou rícino é importante para a utilização em biocombustíveis. Com o objetivo de avaliar seu atual estado foi realizado, em 40 aldeias de influência dos complexos petroleiros Caricare (município Arauquita) e Caño Limón (município de Arauca) em Arauca, na Colômbia, uma coleta de sementes sexual entre dezembro de 2011 e janeiro de 2012, onde há cinco anos a cultura foi estabelecida. Entre as variáveis analisadas foram incluídas dados de passaporte como fonte de coleta, o nome do local, relevo, tipo de solo, entre outros. Para identificar os transectos e registrar dados como localização geográfica e altitude acima do nível do mar foi empregado o GPS Garmin map 76CSx. Aplicou-se a estatística descritiva simples para identificar o conjunto de variáveis com maior variação. Com as variáveis qualitativas mais importantes foi utilizada a análise de tabelas de contingência e contraste com um nível de significância a 5\%, a análise de correspondência múltipla (ACM) e de componentes principais (PCA) para as variáveis quantitativas e qualitativas, e foi realizada análise de classificação a partir de uma matriz de similaridade. A mamona foi encontrada em $25 \%$ das aldeias visitadas. Doze apresentações foram coletadas, quatro delas em Caricare e oito em Caño Limón. Não obstante as condições ambientais serem favoráveis ao seu cultivo, não foram encontradas culturas comerciais nos locais visitados. A ausência de comercialização e equipamentos de grãos para extração do óleo foram o principal fator limitante. Foi criado um jardim com os materiais coletados com a finalidade de uso em pesquisa, criação e promoção.

Palavras-chave: biodiesel, Caricare Caño Limón, mamona, rícino, Ricinus communis L.

\section{Introducción}

El agotamiento de las reservas petrolíferas y el incremento en la demanda de fuentes energéticas confirman que el biodiesel es una de las alternativas más prometedoras en relación a los combus- tibles fósiles. En Colombia, se estima que entre gasolina y diesel (ACPM), representan cerca del $47 \%$ de la demanda total de energía en el sector transporte (Restrepo, et al., 2010). 
Considerado como el mejor para producir biodiesel por ser el único soluble en alcohol sin requerir calor, con el consecuente gasto de energía que exigen otros aceites vegetales (EMBRAPA, 2005), $R$. communis $L$. se cultiva comercialmente en más de quince países, siendo India, China y Brasil los mayores productores (Vieira y Lima, 2008), este último con 149 mil toneladas del grano (CONAB, 2009).

En introducciones de higuerilla colectadas espontáneamente en los Estados de Aragua, Bolívar, Sucre, Lara, en Venezuela, y dos variedades mejoradas del Instituto Nacional de Investigaciones Agrícolas (INIA) - Centro Nacional de Investigaciones Agropecuarias (CENIAP), se encontró un peso promedio de 100 semillas de 29,37 g con un coeficiente de variación de $51,72 \mathrm{~g}$ (Mazzani y Rodríguez, 2009). En Brasil, en un estudio de la variación en semilla de 49 introducciones de una colección de higuerilla, mediante análisis de componentes principales, se encontró una varianza acumulada de $96,54 \%$ en los dos primeros componente principales, observando que el largo y el peso de 100 semillas fueron las características más importantes, con una correlación positiva y significativa entre ellas $(0,96)$ (Figueiredo-Neto et al., 2004).

Para Colombia no se reportan colectas de higuerilla, esta se cultiva como cerco vivo, en huertas caseras o jardines decorativos y aunque regiones como los valles interandinos y llanos orientales poseen condiciones ambientales propicias, no se cultiva a nivel comercial (Ríos et al., 2008). Sin embargo, en el año 2005 en el departamento de Arauca se establecieron cultivos comerciales, por lo que se visitaron 40 veredas del área de influencia de los complejos petroleros de Caricare (municipio de Arauquita) y Caño Limón (municipio de Arauca) en Arauca, Colombia, con el objetivo principal de caracterizar el estado de la especie y colectar germoplasma.

Desde el punto de vista estratégico la colecta de germoplasma de esta especie, tiene entre otros propósitos i) la investigación taxonómica, citogenética y biosistemática, ii) el estudio de la biodiversidad genética y la conservación y, iii) el uso inmediato en los programas de mejoramiento (Bothner y Seberg, 1995).

\section{Materiales y métodos}

La investigación se realizó con la coordinación del proyecto "Caracterización de la producción de higuerilla (Ricinus communis L.) en la zona de Caricare (Municipio de Arauquita) y Caño Limón (Municipio de Arauca) Colombia", con la participación conjunta del Grupo de Investigación en Recursos Fitogenéticos GIRFIN, en la sede Palmira, y la oficina de investigaciones en la sede Orinoquía, de la Universidad Nacional de Colombia.

La colecta de $R$. communis L. consistió en obtener semilla sexual. Esta se realizó entre los meses de diciembre de 2011 y enero de 2012, en 17 veredas del área de influencia del complejo petrolero de Caricare y en 23 veredas del complejo petrolero de Caño Limón en el departamento de Arauca, Colombia (Figura 1). Se realizaron transectos entre las veredas de influencia de cada complejo petrolero (Tablas 1 y 2 ).
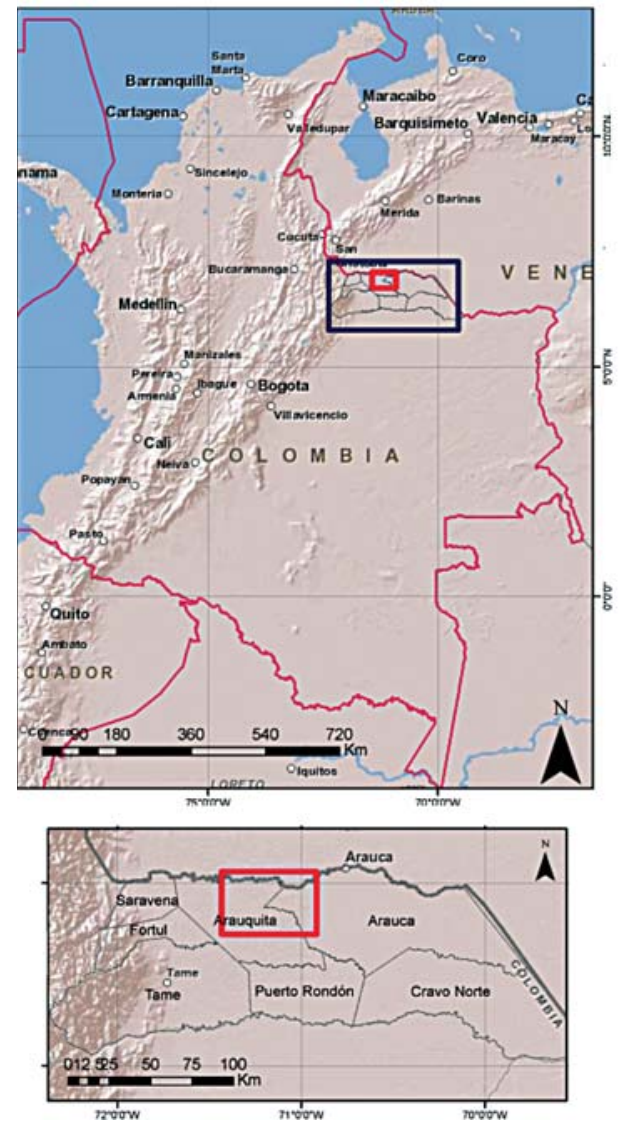

Figura 1 Ubicación geográfica del estudio.

Fuente: BingMaps 2012. Cartografía base Colombia Instituto Geográfico Agustín Codazzi (IGAC, 2006) 
Cada introducción consistió de semillas colectadas completamente al azar entre poblaciones de plantas halladas en cada punto referenciado geográficamente. El número de semillas varió conforme a la disponibilidad de plantas. Se colectaron semillas al menos en el $50 \%$ de las plantas encontradas. Para cada introducción se registraron datos de pasaporte tales como fecha, sitio y fuente de colecta, así como tipo de germoplasma, características de la población, de la semilla, el terreno, el paisaje y el suelo.

Tabla 1. Transectos de la zona de influencia del complejo petrolero de Caricare

\begin{tabular}{|c|c|c|c|}
\hline No & Municipio & Transecto & $\begin{array}{l}\text { Subtotal } \\
\text { veredas }\end{array}$ \\
\hline 1 & Arauquita & $\begin{array}{l}\text { Panamá de Arauca, } \\
\text { El Sinaí, San Juan } \\
\text { de Reinera }\end{array}$ & 3 \\
\hline 2 & Arauquita & $\begin{array}{l}\text { Los Colonos, Santa } \\
\text { Clara, La Reserva }\end{array}$ & 3 \\
\hline 3 & Arauquita & $\begin{array}{l}\text { Los Fundadores, } \\
\text { Playa Rica }\end{array}$ & 2 \\
\hline 4 & Arauquita & $\begin{array}{l}\text { San Isidro, Gaviotas } \\
\text { del Caranal }\end{array}$ & 2 \\
\hline 5 & Arauquita & $\begin{array}{l}\text { Tropicales, } \\
\text { Puerto Rico }\end{array}$ & 2 \\
\hline 6 & Arauquita & $\begin{array}{l}\text { El Carmen, El Futuro, } \\
\text { La Gloria, El Rosal }\end{array}$ & 4 \\
\hline \multirow[t]{2}{*}{7} & Arauquita & Vista Hermosa & 1 \\
\hline & & Total veredas & 17 \\
\hline
\end{tabular}

Datos de ubicación y altura sobre el nivel del mar se registraron mediante un GPS marca Garmin map76CSx. DIVA-GIS software (versión 3.2) y se utilizaron para mapear la colecta de $R$. communis $L$.
Tabla 2. Transectos de la zona de influencia del complejo petrolero de Caño Limón

\begin{tabular}{|c|c|c|c|}
\hline No & Municipio & Transecto & $\begin{array}{l}\text { Subtotal } \\
\text { veredas }\end{array}$ \\
\hline 1 & Arauquita & $\begin{array}{l}\text { La Pesquera, } \\
\text { Las Acacias, } \\
\text { Puerto Nuevo }\end{array}$ & 3 \\
\hline 2 & Arauquita & La Pica, Las Bancas & 2 \\
\hline 3 & Arauquita & Mataoscura, La Osa & 2 \\
\hline 4 & Arauca & $\begin{array}{l}\text { Caño Limón, El Final, } \\
\text { Nubes A, Nubes B, } \\
\text { El Sinaí, Todos Los } \\
\text { Santos }\end{array}$ & 6 \\
\hline 5 & Arauquita & $\begin{array}{l}\text { La Arenosa, } \\
\text { Los Angelitos A }\end{array}$ & 2 \\
\hline 6 & Arauquita & $\begin{array}{l}\text { Nueva Jerusalen, } \\
\text { Los Masaguaros, } \\
\text { La Maporita, } \\
\text { Los Laureles, } \\
\text { Gran Bretaña }\end{array}$ & 5 \\
\hline \multirow[t]{2}{*}{7} & Arauquita & $\begin{array}{l}\text { La Reinera, Caño } \\
\text { Arenas, Santa } \\
\text { Bárbara, La Arenosa* }\end{array}$ & 3 \\
\hline & & Total veredas & 23 \\
\hline
\end{tabular}

*La vereda de La Arenosa es dividida por el Caño Limón, se cuenta como una sola.

Entre las variables cuantitativas para las introducciones se incluyó altura sobre el nivel del mar (asnm), y en las semillas, peso de 10 semillas (P10 semillas en g), ancho de semilla (AS, promedio de 10, en $\mathrm{cm}$ ), largo de semilla (LS, promedio de 10, en $\mathrm{cm}$ ), peso total (peso total introducción), número de semillas ( $\mathrm{N}$ semillas). Entre las variables cualitativas se incluyó fuente de colecta (fuente), nombre común (nombre), usos (uso), tipo de vegetación asociada (vegetación) y drenaje del suelo (drenaje). 


\section{Análisis de resultados}

Se realizó un análisis estadístico descriptivo simple para identificar el conjunto de variables con mayor variación. Los descriptores cuantitativos fueron estudiados mediante análisis de componentes principales (ACP). La contribución de los descriptores entre las introducciones fue determinada a través de vectores propios derivados de la matriz de correlación entre los 11 caracteres. Grupos de introducciones semejantes fueron identificados mediante análisis de clasificación jerárquica ascendente (CJA), utilizando el criterio de varianza mínima de Ward entre introducciones.

Para el análisis de la variabilidad en variables cualitativas de mayor importancia se realizó un análisis de tablas de contingencia contrastando a un nivel de significancia del $5 \%$, análisis de correspondencia múltiple (ACM) y de componentes principales (ACP) para variables cuantitativas y cualitativas. Se realizó un análisis cluster o de clasificación a partir de una matriz de similitud.

\section{Resultados y discusión}

Se encontró higuerilla creciendo espontáneamente en el $25 \%$ del total de las veredas visitadas. En la zona de Caricare el 3\% de las veredas demostró presencia de la especie, mientras que en las veredas de la zona de Caño Limón fue del 30\%. Del total de veredas visitadas se colectaron cuatro introducciones de semillas de higuerilla en el área de influencia de Caricare (Tabla 3) y ocho en Caño Limón (Tabla 4), todas de origen silvestre (Figura 2, números son consecutivos de las introducciones). Se encontró en grupos de dos a 12 plantas, entre los 137 a 175 $\mathrm{msnm}, 30^{\circ} \mathrm{C}$ de temperatura, en planicies con pendientes de $0 \%$ a $5 \%$, en espacios abiertos a la luz del sol, en bordes de formaciones boscosas, caminos, matorrales de baja altura y pasturas principalmente; con suelos franco limosos, grisáceos, bien drenados, de escasa erosión, con fertilidad moderada y de mediano a bajo contenido de materia orgánica. En cuatro de las localidades colectadas se encontró como uso en conservación, sombrío para huertos, aceite para combustible, medicina y cosmético. La quema de la semilla ahuyenta zancudos, y se usa como purgante en animales como cerdos y perros. No se observaron enfermedades en las plantas de donde provinieron las semillas colectadas.

Tabla 3. Número de semillas colectadas en la zona de Caricare

\begin{tabular}{cllccc}
\hline Transecto & \multicolumn{1}{c}{ Vereda } & $\begin{array}{c}\text { Número } \\
\text { introducción* }\end{array}$ & $\begin{array}{c}\text { Número } \\
\text { de semillas }\end{array}$ & $\begin{array}{c}\text { Peso 10 } \\
\text { semillas (g) }\end{array}$ & $\begin{array}{c}\text { Número } \\
\text { plantas }\end{array}$ \\
\hline 2 & Los colonos & RC170-007-004 & 350 & 1,33 & 2 \\
\hline 3 & Playa Rica & RC170-007-003 & 175 & 1,12 & 3 \\
\hline 4 & San Isidro & RC170-007-001 & 30 & 1,01 & 2 \\
\hline 4 & San Isidro & RC170-007-002 & 827 & 1,21 & 5 \\
\hline
\end{tabular}

${ }^{*} \mathrm{RC} 170-007-004$ es Ricinus communis L. número internacional para Colombia - número nacional para la región de Orinoquía - consecutivo de colecta. 
Con las semillas colectadas se estableció un banco de germoplasma en predios de la Universidad Nacional de Colombia en Palmira, Valle del Cauca. Las introducciones se sembraron en un diseño completamente al azar en cuatro repeticiones. Se espera que sirva para adelantar investigaciones morfológicas, reológicas y genéticas.

Tabla 4. Número de semillas colectadas en la zona de Caño Limón

\begin{tabular}{cllccc}
\hline Transecto & Vereda & $\begin{array}{c}\text { Número } \\
\text { introducción* }\end{array}$ & $\begin{array}{c}\text { Número } \\
\text { de semillas }\end{array}$ & $\begin{array}{c}\text { Peso 10 } \\
\text { semillas (g) }\end{array}$ & $\begin{array}{c}\text { Número } \\
\text { plantas }\end{array}$ \\
\hline 1 & Las Acacias & RC170-007-006 & 294 & 2,82 & 6 \\
\hline 1 & Las Acacias & RC170-007-007 & 195 & 2,90 & 7 \\
\hline 3 & La Ossa & RC170-007-005 & 290 & 1,25 & 12 \\
\hline 4 & El final & RC170-007-008 & 100 & 2,82 & 4 \\
\hline 4 & Nubes A & RC170-007-009 & 180 & 0,96 & 4 \\
\hline 5 & La Arenosa & RC170-007-012 & 210 & 2,99 & 3 \\
\hline 7 & Caño Arenas & RC170-007-010 & 59 & 1,13 & 1 \\
\hline 7 & Cogollal & RC170-007-011 & 216 & 1,38 & 3 \\
\hline
\end{tabular}

*RC170-007-006 es Ricinus communis L. número internacional para Colombia - número nacional para la región de Orinoquía - consecutivo de colecta.

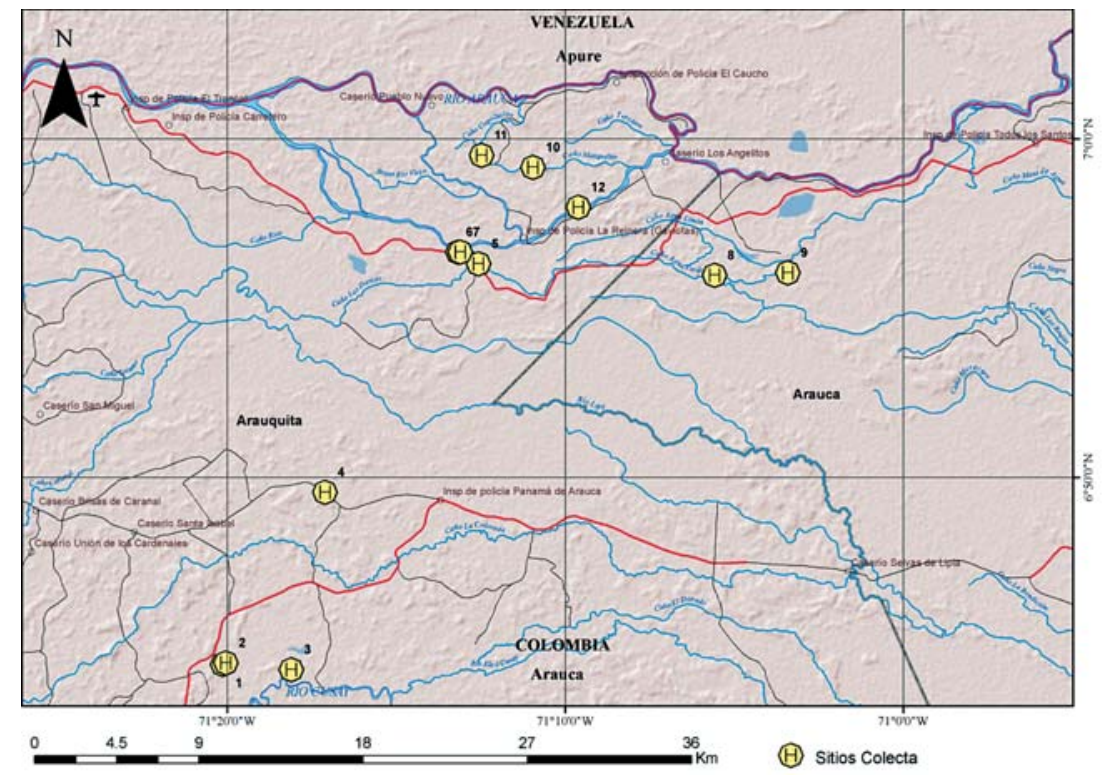

Figura 2. Distribución espacial de las introducciones colectadas.

Fuente: BingMaps 2012. Cartografía base Colombia. Instituto Geográfico Agustín Codazzi (IGAC, 2006) 
Los frutos colectados generalmente poseían tres semillas. Eventualmente se observaron frutos con más de tres semillas. La camada con acúleos que cubre la semilla se desprendió manualmente con relativa facilidad, lo que constituye una característica de interés en higuerilla (Soares y de Souza, 2005), sin embargo sobre esta propiedad influyen otras características ambientales como la baja humedad relativa al momento de la cosecha.

El tegumento de las semillas colectadas presentó diferencias en colores y diseños gráficos (Figura 3). Los colores de fondo variaron entre gris y marrón, mientras las líneas variaron entre rojas y marrón oscuro.

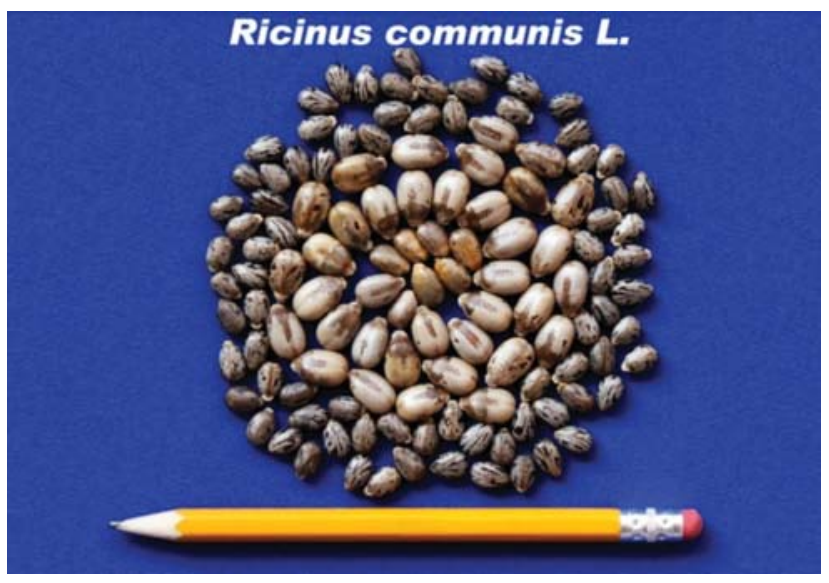

Figura 3. Semillas colectadas en Arauca y Arauquita, Arauca, Colombia

La estadística descriptiva de los seis descriptores cuantitativos demostró que existe variabilidad entre las 12 introducciones colectadas (Tabla 5). La variación más importante fue encontrada para Número de semillas y peso total de la muestra, descriptores que se encuentran asociados con la disponibilidad de semilla en las localidades donde se hallaron plantas de higuerilla.

Tabla 5. Estadística descriptiva de los descriptores cuantitativos en las introducciones de higuerilla (Ricinus communis L.) en 40 veredas del departamento de Arauca, Colombia.

\begin{tabular}{lccccc}
\hline \multicolumn{1}{c}{ Descriptor } & Media & $\begin{array}{c}\text { Desviación } \\
\text { estándar }\end{array}$ & $\begin{array}{c}\text { Coeficiente } \\
\text { de variación }\end{array}$ & Mínimo & Máximo \\
\hline Altitud (msnm) & 153,31 & 11,11 & 7,25 & 135,00 & 175,00 \\
\hline Peso 10 semillas (g) & 1,74 & 0,84 & 48,79 & 0,96 & 2,99 \\
\hline Largo semilla (cm) & 0,89 & 0,27 & 31,36 & 0,11 & 1,33 \\
\hline Ancho semilla (cm) & 0,62 & 0,07 & 11,70 & 0,57 & 0,83 \\
\hline Peso total (g) & 40,83 & 2,17 & 73,59 & 2,95 & 100,02 \\
\hline Número de semillas & 243,92 & 206,57 & 84,69 & 30,00 & 827,00 \\
\hline
\end{tabular}

El peso y el largo de semilla también presentaron variaciones importantes. El peso promedio para 10 semillas fue de 1,74 g con un coeficiente de variación de $48,79 \%$ para largo de semilla el promedio fue de 0,89 cm con un coeficiente de variación de $31,36 \%$ indicando gran variación para estos dos descriptores. El ancho de semilla presentó bajos valores de variación. En un trabajo similar se encontraron pesos promedio en 100 semillas de 29,37 g con valor mínimo de $14,76 \mathrm{~g}$ y máximo de $67,94 \mathrm{~g}$ con coeficiente de variación de 51,72\%, mientras que para el largo de semilla se encontró una media de $1,35 \mathrm{~cm}$ con valor mínimo de $1,07 \mathrm{~cm}$ y máximo de $1,94 \mathrm{~cm}$ (Manzzani \& Rodríguez, 2009). Aunque se observaron coeficientes de variación inferiores con respecto al trabajo reportado, los valores máximos y mínimos para descriptores de semilla son superiores y de interés para posteriores estudios. 
De acuerdo al análisis de componentes principales (Tabla 6) el primer componente explicó el $35,08 \%$ de la variabilidad y el segundo componente el $21,49 \%$ y el tercero el $18,02 \%$, acumulando el $74,58 \%$ de la variabilidad total. Al primer componente se asociaron variables de tipo de uso, vegetación y ancho de semilla; al segundo componente se asociaron nombre común y drenaje del suelo; entre tanto al tercer componente se asociaron peso total, número de semillas y peso de 10 semillas (Figura 4).

Tabla 6. Autovalores y variabilidad explicada por los componentes principales categóricos de 11 descriptores de las introducciones de higuerilla (Ricinus communis L.) en Arauca, Colombia.

\begin{tabular}{cccc}
\hline $\begin{array}{c}\text { Compo- } \\
\text { nente }\end{array}$ & Valor & Proporción & $\begin{array}{c}\text { Proporción } \\
\text { acumulada }\end{array}$ \\
\hline 1 & 3,858 & 35,08 & 35,08 \\
\hline 2 & 2,363 & 21,49 & 56,56 \\
\hline 3 & 1,982 & 18,02 & 74,58 \\
\hline
\end{tabular}

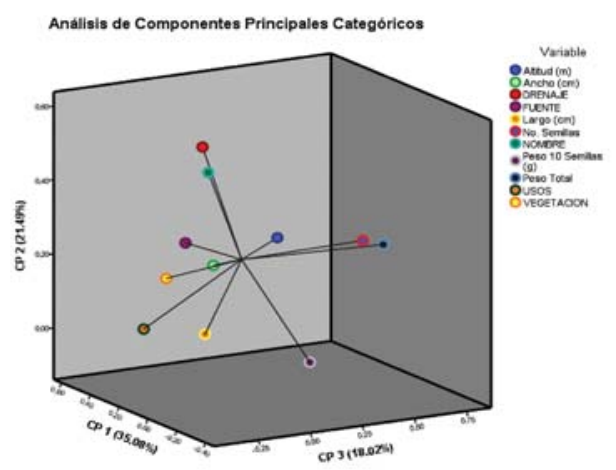

Figura 4. Distribución de los descriptores por su asociación a los tres componentes principales categóricos.

Al 26\% de la distancia, la CJA presentó la formación de 4 grupos de introducciones (Figura 5) descritos así:

Grupo I: se incluyen en este grupo las introducciones 5 y 8 , colectadas en la Ossa y El Final respectivamente, correspondientes a los transectos 3 y 4 en Caño Limón, caracterizándose por no presentar ningún tipo de uso, halladas en bordes de pastizales, de semillas medianas, en suelos escasamente drenados, conocidas principalmente por los agricultores vecinos con el nombre de tártago. Correspondieron a las más bajas altitudes sobre el nivel del mar. El número total de semillas colectadas en promedio indica la moderada disponibilidad de semillas en los sitios de colecta.

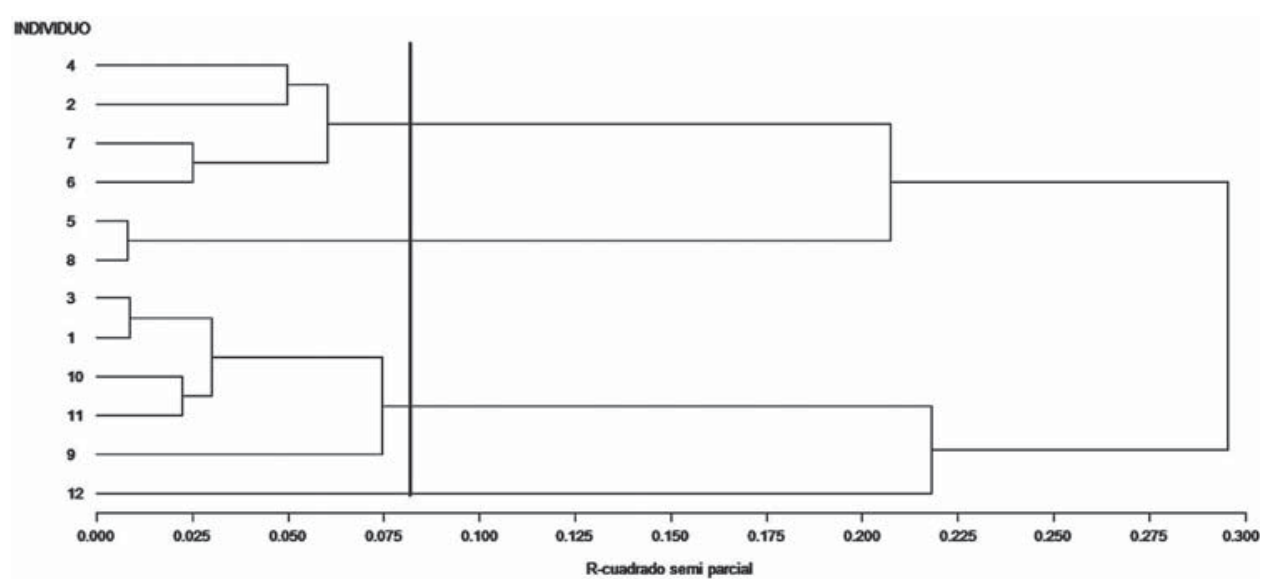

Figura 5. Clasificación jerárquica ascendente de 12 introducciones de la colección de higuerilla con base a 11 descriptores de datos de pasaporte en Arauca, Colombia. 
Grupo II: es el más numeroso, constituido por 1, 3, 9, 10 y 11, colectadas en San Isidro (transecto 4) y Playa Rica (transecto 3) en Caricare, y en Las Nubes A (transecto 4), Caño Arenas y El Cogollal (transecto 7) en Caño Limón, respectivamente; se caracterizan por presentar uso como aceite, se las encontró en bordes de pastizales, bosques y matorrales de baja altura indicando alguna tolerancia a la asociación con otras plantas, sus semillas son medianas pero livianas, presentan el más bajo promedio de total de semillas colectadas indicando una baja disponibilidad de su semilla en las localidades colectadas destacándose las introducciones 1 y 10 como las más escasas de toda la colecta. Este grupo se encontró en suelos preferiblemente drenados. Entre los agricultores se le conoce por el nombre local de higuerilla.

Grupo III: constituido por las introducciones 2, 4, 6 y 7, colectadas en San Isidro (transecto 4) y los colonos (transecto 2) en Caricare, y en Las Acacias (transecto 1) en Caño Limón, respectivamente. Se caracterizan por ubicarse en las más elevadas alturas sobre el nivel del mar para la zona de estudio, suelos bien drenados y el mayor número de semillas colectadas por localidad lo que las hace muy disponibles, poseen las semillas relativamente pequeñas pero con mejores pesos promedio para semilla. Solo la introducción número 4 reportó uso como medicinal.

Grupo IV: correspondió a la introducción número 12, colectada en La Arenosa (transecto 5) en Caño Limón; se distinguió por tener un uso en aceite, hallada en un sendero relativamente boscoso y en suelos bien drenados indicando su capacidad de tolerar el sombrío. Presenta las semillas más grandes y más pesadas haciéndola un material de interés para su evaluación en el contenido de aceite.

\section{Conclusiones}

Se encontró higuerilla creciendo espontáneamente en el $25 \%$ del total de las veredas visitadas. No se observaron prácticas culturales con la especie. Aunque se cuenta con las condiciones ambientales propicias para la siembra de la especie, la ausencia de tecnología adecuada para la extracción del aceite y del mercado limitan su desarrollo. Las introducciones colectadas son de naturaleza silvestre y presentan descriptores de interés productivo. Se requiere ampliar la colecta y adelantar evaluaciones genéticas del material colectado con fines de uso en la industria de los biocombustibles.

\section{Agradecimientos}

A la Fundación El Alcaraván, a la Universidad Nacional de Colombia sedes Orinoquía y Palmira, a las autoridades municipales y a los agricultores de las veredas visitadas en los municipios de Arauca y Arauquita en el departamento de Arauca, Colombia.

\section{Literatura citada}

1. Bothmer, R. von \& Seberg, O. (1995). Strategics for the collecting of wild species. In: Collecting plant genetic diversity technical guidelines (Eds. Guarino, L.; Ramanatha Rao, V. \& Reid, R.). CAB International, UK. P. 93

2. CONAB. (2009). Companhia Nacional de Abastecimento. Acompanhamento da safra brasileira de graos 2009/2010. On line: http://www.conab.gov.br/conaweb Fecha de acceso: 5 de octubre de 2010.

3. EMBRAPA (2005). Comportamento de mamoneira sob encharcamento do solo. Boletin de pesquisa e desenvolvimento No $57 ; 16 \mathrm{pp}$.

4. Figueiredo-Neto, A.; F de Assis; C. Almeida; J. P. G de Gouveia; M. B. M. Nóbrega; R. M. Carneiro \& J. P. Pedroza. (2004). Divergência genética em acessos de mamona (Ricinus communis L.) baseada nas características das sementes. Revista de Biologia e Ciências da Terra 4 (2): $15-20$. 
5. Mazzani, E. \& Rodríguez, E. (2009). Estudio de la variabilidad presente en germoplasma de tártago (Ricinus communis L.) en cuanto a racimos, frutos y semillas. Centro Nacional de Investigaciones Agropecuarias, Instituto Nacional de Investigaciones Agrícolas. Estado de Aragua, Venezuela.

6. Restrepo, H. J. F.; Salazar, S. G. \& Aristizabal, M. M. (2010). Alternativas de producción del higuerillo (ricinus communis L.) con fines de extracción de aceites para biodiesel y la industria oleoquímica como estrategia para el fortalecimiento del departamento de Caldas. En: Congresso brasileiro de mamona, 4 y Simposio internacional de oleaginosas energéticas, 1, 2010, Joao Pessoa. Inclucao Social e Energia: Anais... Campina Grande: EMBRAPA Algodao, 2010, p. 1-6
7. Ríos, G. G.; Vásquez, G. L. A.; Díaz, A. R. \& Domínguez, V. A. (2008). Limitaciones y potencialidades de los sistemas de producción de higuerilla (Ricinus communis L.). En: Higuerilla: una alternativa productiva, energética y agroindustrial para Colombia.

8. Soares, S. L. \& de Souza G. T. M. (2005). Curso sobre el cultivo de higuerilla. Embrapa, Brasil - INIA, Venezuela.

9. Vieira, R. M. \& Lima, E. F. (2008). Importancia socioeconómica e melhoramento genético de mamoneira no Brasil. In Queiróz, M. A. de; Goedert, C. O.; Ramos, S. R. R. (ed.). Recursos genéticos e melhoramento de plantas para o nordeste brasileiro. On line: http://www.cpatsa.embrapa.br Fecha de acceso: 5 de octubre de 2010. 